\title{
Bloqueo continuo erector de la espina (ESP) para cirugía torácica resectiva por toracotomía
}

\section{Continuous erector spinae plane block (ESP) for thoracotomy in thoracic resective surgery}

\author{
Mario Sepúlveda', Martín Friedman'1, Rodrigo Villarroel ${ }^{2}$, Glenda Sánchez ${ }^{3}$, Roberto Coloma ${ }^{1, *}$, Sebastián Merino1 \\ Anestesiólogo, Hospital Sótero del Río. \\ 2 Cirujano de Tórax, Hospital Sótero del Río. \\ 3 Enfermera Universitaria, Unidad del dolor, Hospital Sótero del Río.
}

Conflicto de intereses: los autores no declaran conflictos de interés en el estudio.

Fuente de financiamiento: no existe financiamiento de ninguna identidad.

Fecha de recepción: 31 de julio de 2021 / Fecha de aceptación: 15 de agosto de 2021

\begin{abstract}
Introduction: Post-operative pain in resective thoracic surgery is one of the most intense. Among the classic analgesic alternatives described for this surgery are thoracic epidural analgesia and paravertebral block. The erector spinae plane block (ESP) consists of an interfascial block that is performed at the level of the transverse process of T5. The main objective of the present study is to present a series of cases that show the analgesic efficacy of ESP block both intra and postoperatively in patients undergoing resective thoracic surgery. Material and Methods: With a $50 \mathrm{~mm}-100 \mathrm{~mm}$ Pajunk needle and under ultrasound vision, interfascial plane was identified at the level of the transverse process of T5 and a single injection of Levobupivacaine $0.25 \% 20$ cc was performed and a catheter was installed for continuous infusion. Results: 7 patients underwent thoracic surgery, after performing continuous ESP block with catheter installation. We used $0.1 \%$ Bupivacaine with a continuous infusion of $2 \mathrm{~mL} / \mathrm{h}$, programmed intermittent boluses of $15 \mathrm{~mL}$ every 4 hours, and boluses of $5 \mathrm{~mL}$ controlled by the patient in case of pain and with a 1-hour blockade. Pain in all patients remained below 3 points on the visual analog scale during the 48-72 postoperative hours, and without the need for rescue opioid analgesics. Discussion: continuous ESP block is a great analgesic alternative for resective thoracic surgery.
\end{abstract}

Key words: Erector spinae plane block, thoracotomy, thoracic surgery, pulmonary lobectomy.

\section{RESUMEN}

Introducción: El dolor posoperatorio en cirugía torácica resectiva por toracotomía es uno de los más intensos posquirúrgicos. Entre las alternativas analgésicas clásicas descritas para esta cirugía están la analgesia peridural torácica y el bloqueo paravertebral. El bloqueo del erector de la espina (ESP) consiste en un bloqueo interfascial que se realiza a nivel de la apófisis transversa de T5. El objetivo principal del presente estudio es presentar una serie de casos que muestran la eficacia analgésica del bloqueo ESP tanto en el intra como en el posoperatorio de los pacientes sometidos a cirugía torácica resectiva por toracotomía. Material y Métodos: Con aguja Pajunk $50 \mathrm{~mm}$ - $100 \mathrm{~mm}$ y bajo visión con ultrasonido se identificó plano interfascial a la altura de apófisis transversa de T5 y se realizó inyección única de levobupivacaína 0,25\% 20 cc e instalación de catéter para infusión continua posoperatoria. Resultados: Siete pacientes fueron sometidos a cirugía torácica por toracotomía, previa realización de Bloqueo ESP continuo con instalación de catéter. Utilizamos bupivacaína $0,1 \%$ con infusión continua $2 \mathrm{~mL} / \mathrm{h}$, bolos intermitentes programados de $15 \mathrm{~mL}$ cada $4 \mathrm{~h}$ y bolos de $5 \mathrm{~mL}$ controlados por el paciente en caso de dolor y con bloqueo de $1 \mathrm{~h}$. El dolor en todos los 
pacientes se mantuvo por debajo de 3 puntos de la escala visual análoga durante las 48-72 h posoperatorias, y sin requerimiento de analgésicos opioides de rescate. Discusión: El bloqueo ESP continuo es una gran alternativa analgésica para cirugía torácica resectiva por toracotomía.

Palabras clave: Bloqueo erector de la espina, toracotomía, cirugía de tórax, lobectomía pulmonar.

\section{Introducción}

E dolor posoperatorio en cirugía torácica resectiva por toracotomía es uno de los más intensos posquirúrgicos, debido a múltiples factores relacionados con la cirugía y el período peri operatorio. Retracciones costales, resección pulmonar, daño de nervios intercostales, entre otros, determinan un posoperatorio complejo, con una lenta recuperación de la función pulmonar y una mayor tasa de complicaciones, como mayor incidencia de atelectasias y tos inefectiva, que podría traer mayor número de complicaciones infecciosas en el caso de no contar con una analgesia y kinesioterapia adecuada[1],[2]. Un manejo precoz y dirigido de este dolor se relacionará con una mejor evolución en el posoperatorio, tolerancia a kinesioterapia precoz, menos días de hospitalización y menor riesgo de dolor crónico posquirúrgico[1],[2].

Entre las alternativas analgésicas clásicas descritas para la cirugía por toracotomía encontramos la analgesia sistémica intravenosa, peridural torácica, bloqueo paravertebral, o un bloqueo de los nervios intercostales, cada uno de los cuales presenta beneficios y riesgos conocidos. Si hablamos de un "Gold Standard" probablemente la mayoría de los centros y guías consideran la analgesia peridural y paravertebral[3], las cual dan un bloqueo sensitivo eficaz y adecuado - uni o bilateral - a toda la pared torácica, sin embargo, teniendo ciertos efectos adversos no deseables como riesgo de punción dural con la consecuente cefalea, lesión medular, hipotensión, prurito, retención urinaria y bloqueo motor con dificultad en la deambulación posoperatoria, entre otras[3],[4].

Desde el 2016 contamos con otra alternativa analgésica, cuando Forero y cols.[5], describen el bloqueo del erector de la espina (ESP) en cadáveres inicialmente y luego en pacientes con dolor crónico[6]. Éste consiste en un bloqueo interfascial que se realiza a nivel de la apófisis transversa de T5, con un abordaje por la región torácica posterior ipsilateral al hemitórax de la cirugía (Figura 1). Es descrito como relativamente fácil y seguro bajo ecografía, con bajo riesgo de hematoma y sin invasión del neuroeje.

Los primeros estudios cadavéricos y luego en pacientes voluntarios bajo marcación y RNM han mostrado una distribución craneocaudal del anestésico local con un "spread" entre T2T9[5],[7] y un bloqueo de la rama dorsal del nervio espinal en todos los casos, y aún en discusión de la rama ventral[8].

Dos metaanálisis publicados en 2020 muestran moderada calidad de evidencia de disminución del dolor posoperatorio con el bloqueo ESP para diversas cirugías, incluyendo cirugía torácica y de mama, demostrando igual calidad analgésica que bloqueo paravertebral[9],[10]. Otro estudio randomizado y controlado de Yao y cols., del año 2020, demostró con un cuestionario la calidad de analgesia y satisfacción del paciente luego de una videotoracoscopía con el bloqueo ESP[11]. Wany y cols.[12], publicaron en 2019 un estudio que demuestra mejor calidad de analgesia y disminución de consumo de opioides al usar bloqueo ESP vs infiltración de herida operatoria en esofagectomía por toracotomía. Sin embargo, más estudios son

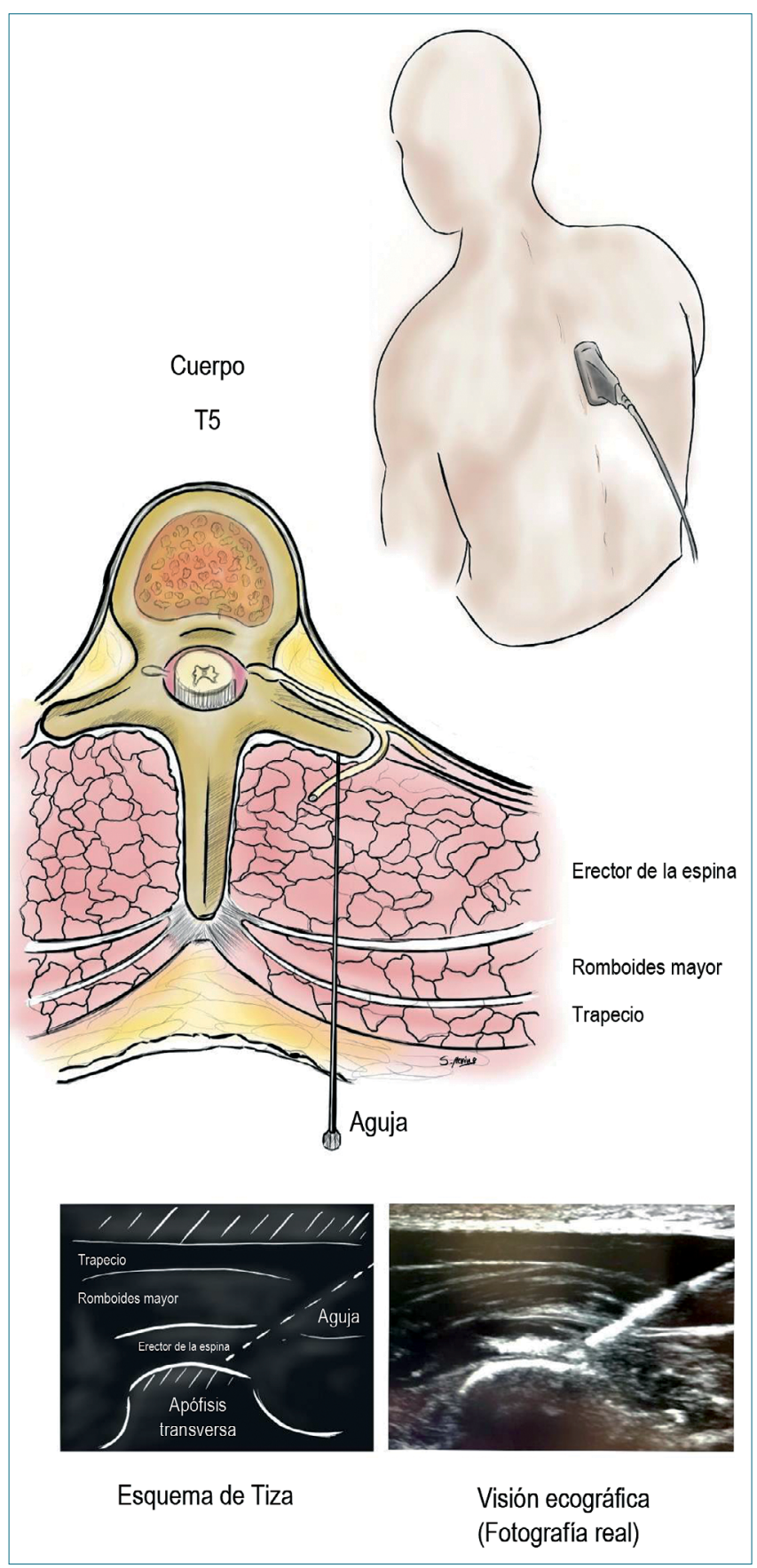

Figura 1. Bloqueo ESP. 
necesarios para comprobar evidencia del bloqueo ESP como método analgésico después de cirugía resectiva pulmonar por toracotomía.

\section{Objetivos}

El objetivo principal del presente estudio es presentar una serie de casos que muestran la eficacia analgésica del bloqueo ESP y su impacto tanto en el intra como en el posoperatorio de los pacientes sometidos a cirugía torácica resectiva por toracotomía. La evaluación del dolor y consumo de opioides de los pacientes fue realizada por parte del equipo del dolor agudo posoperatorio del hospital Sótero del Río, conformado por una enfermera de dolor agudo y un anestesiólogo, con el fin de lograr estandarizar y expandir el uso de esta técnica analgésica de forma rutinaria.

Como objetivo secundario mostrar que facilita la rehabilitación precoz y el alta hospitalaria sin reingreso por dolor.

\section{Material y Métodos}

Consentimiento informado fue solicitado a todos los pacientes previo a la cirugía y realización de bloqueo ESP. El estudio fue aprobado por el comité de ética del hospital docente asistencial Dr. Sótero del Río.

El enrolamiento para el estudio fue ofrecido a pacientes mayores de 18 años que se realizarían una cirugía de tórax resectiva por toracotomía o videotoracoscopía con alto riesgo de conversión, y que aceptaran la realización de un bloqueo ESP con la instalación de un catéter continuo.

Criterios de exclusión: rechazo del paciente a la realización del bloqueo y/o instalación del catéter. Uso crónico o historia de abuso de opioides por parte del paciente.

\section{Bloqueo ESP e inserción catéter en el plano interfascial}

Los pacientes fueron posicionados en decúbito lateral o sentados, con acuerdo a la comodidad del paciente y del operador.

El procedimiento se realizó bajo monitorización estándar con electrocardiografía continua, oximetría de pulso y presión arterial no invasiva, y sedación superficial con midazolam 1-2 mg titulada para manejo de ansiedad de los pacientes, con apo- yo de $\mathrm{O}_{2}$ suplementario por mascarilla Campbell. El bloqueo ESP y la instalación del catéter fueron realizados por 2 anestesiólogos del Servicio del Hospital Sótero del Río, asistidos por un técnico en anestesia.

El plano interfascial del lado ipsilateral a la cirugía fue identificado usando un transductor de ultrasonido linear $13 \mathrm{MHz}$ 38-mm en visión en eje longitudinal, a la altura de apófisis transversa de T5. Para asegurar una buena imagen con el ultrasonido y conociendo los reparos anatómicos clásicos, realizábamos un barrido hacia medial visualizando lámina y estructuras musculares clásicas (trapecio, romboide, músculo erector de la espina), y hacia lateral visualizando estructuras óseas compatibles con las costillas.

El sitio a puncionar fue aseptizado con gluconato de clorhexidina, cubierto con un paño fenestrado y se administró anestesia local con lidocaína $2 \%$ en piel y tejido subcutáneo. Con aguja Pajunk 50 mm - 100 mm (de acuerdo con paciente y preferencia de anestesiólogo) y bajo visión con ultrasonido se identificó plano interfascial a la altura de apófisis transversa de T5, se aspiró con la jeringa con el anestésico local para descartar inyección intravascular y se realizó inyección única de levobupivacaína 0,25\% 20 cc, con visualización de disección de planos por el medicamento e instalación de catéter para infusión continua posoperatoria. El catéter fue insertado $1 \mathrm{~cm}$ distal a la punta de la aguja bajo visualización por ultrasonido.

\section{Resultados}

Este estudio incluyó 7 pacientes sometidos a cirugía resectiva pulmonar por toracotomía. Las características y comorbilidades de los pacientes sometidos a cirugía torácica se muestran en las Tablas 1 y 2.

A pesar de la poca información respecto a regímenes de programación de "Patient controlled analgesia" (PCA) para catéteres ESP, utilizamos bupivacaína 0,1\%, con una baja cuantía de infusión continua ( $2 \mathrm{~mL} / \mathrm{h}$ ), principalmente para evitar eventos de oclusión del catéter. Programamos bolos intermitentes programados de $15 \mathrm{~mL}$ cada $4 \mathrm{~h}$. Bolos de $5 \mathrm{~mL}$ controlados por el paciente en caso de dolor y con bloqueo de $1 \mathrm{~h}$.

La evaluación del dolor en todos los pacientes se mantuvo por debajo de 3 puntos de la escala visual análoga durante las 48-72 h posoperatorias siguientes, en las cuales el paciente se mantuvo hospitalizado y sin requerimiento de analgésicos opioides de rescate adicionales durante este período (Tabla 3).

\begin{tabular}{ccccc}
\hline \multicolumn{7}{c}{ Tabla 1. Características pacientes sometidos a cirugía torácica } \\
\hline Paciente $(\mathbf{n})$ & Género & Edad (años) & Peso (kg) & Altura (cm) \\
1 & Mujer & 73 & 58 & 165 \\
2 & Hombre & 55 & 80 & 179 \\
3 & Mujer & 63 & 63 & 161 \\
4 & Mujer & 68 & 60 & 15 \\
5 & Mujer & 40 & 82 & 165 \\
6 & Mujer & 60 & 115 & 159 \\
7 & Hombre & 53 & 75,4 & 187 \\
& Promedio & 58,9 & & 167,3 \\
\hline
\end{tabular}




\begin{tabular}{ccccc}
\multicolumn{5}{c}{ Tabla 2. Comorbilidades pacientes sometidos a cirugía } \\
torácica
\end{tabular}

En todos los pacientes se pudo realizar kinesioterapia en el primer día posoperatorio sin complicaciones. No hubo reingresos al hospital por dolor.

\section{Discusión}

El bloqueo ESP es un bloqueo interfascial descrito por Forero y cols.[5] en 2016, en el cual se administra anestésico local en relación al músculo erector de la columna.

Nuestros reparos anatómicos y ecográficos serán la apófisis transversa de T5 y el grupo muscular trapecio, romboides y erector de la espina.

De acuerdo con estudios con cadáveres y resonancia magnética, dosis que varían entre $20-30 \mathrm{~mL}$ tendrían una importante diseminación cefalocaudal entre T2 y T10[7],[8]. Distintos volúmenes podrían determinar una diseminación más extensa, por lo que la tendencia es a utilizar volúmenes mayores.

Forero y cols.[6] informaron que el bloqueo ESP guiado por ultrasonido proporcionó un bloqueo sensorial adquirido de T3 a T9 sobre el tórax posterior y de T3 a T6 sobre el tórax anterolateral después de la inyección de ropivacaína al 0,5\% (20 ml) en el nivel de T5, y describió que éste era una buena opción para la analgesia después de la lobectomía por videotoracoscopía. Por ello podría ser una alternativa analgésica válida para el manejo del dolor posoperatorio por toracotomía, como lo hemos visto en los casos anteriormente expuestos.

Si bien respecto al bloqueo de nivel de la rama dorsal hay consenso respecto a su efectividad, hay diversas teorías y aún es controversial su efecto a nivel de la rama ventral del nervio espinal.

Ivanusic y cols.[8] un estudio cadavérico para investigar el mecanismo de acción para el bloqueo erector de la espina, donde solo se presenta marcación de la rama dorsal del nervio espinal.

En la misma búsqueda de entender más respecto al mecanismo y cobertura del bloqueo, Schwartzmann y cols.[7] demostraron mediante un estudio con resonancia magnética la diseminación del anestésico local en 6 pacientes voluntarios con dolor, encontrando que el anestésico local alcanzó los espacios intercostales y los agujeros neurales en todos los pacientes, pero con una extensión variable. Además, se diseminó extensamente dentro de los músculos erectores de la columna. El bloqueo sensorial que se logró fue tanto en dermatomas ven-

\begin{tabular}{ccccc}
\multicolumn{5}{c}{ Tabla 3. Evaluación dolor por escala visual análoga $\mathbf{1}$ as $\mathbf{7 2} \mathbf{~}$} \\
\hline $\begin{array}{c}\text { Paciente } \\
\text { (n) }\end{array}$ & EVA $\mathbf{2 4} \mathbf{h}$ & EVA $\mathbf{4 8} \mathbf{h}$ & EVA $\mathbf{7 2} \mathbf{~}$ & $\begin{array}{c}\text { Morfina } \\
\text { rescate }\end{array}$ \\
1 & 2 & 2 & Alta & No \\
2 & 0 & 3 & Alta & No \\
3 & 1 & 4 & 0 & $3 \mathrm{mg}$ \\
4 & 0 & 2 & Alta & No \\
5 & 2 & 3 & 1 & $3 \mathrm{mg}$ \\
6 & 0 & 3 & 2 & No \\
7 & 1 & 0 & Alta & No \\
\hline
\end{tabular}

trales como dorsales en todos los pacientes, aunque su extensión fue variable.

Para nuestra serie de casos expuesta, suponemos que la presente técnica bloquea tanto rama dorsal como rama ventral del nervio espinal, a través del espacio intercostal y el espacio paravertebral torácico.

Aunque el bloqueo ESP en la actualidad cuenta con gran popularidad, requiere de más estudios controlados y randomizados para demostrar su efectividad en el manejo del dolor posoperatorio de la cirugía resectiva pulmonar por toracotomía. Además, su mecanismo de acción no es completamente conocido y existen múltiples teorías respecto a las cuales no ahondaremos. No es nuestra intención demostrar superioridad del bloqueo ESP sobre los gold estándar, como son la analgesia peridural y el bloqueo paravertebral[3], pero sí mostrar los buenos resultados en un grupo reducido de pacientes y la viabilidad y gran alternativa de la presente técnica analgésica para cirugía torácica resectiva por toracotomía.

\section{Referencias}

1. Richardson J, Sabanathan S, Shah R. Post-thoracotomy spirometric lung function: the effect of analgesia. A review. J. Cardiovasc Surg. 1999; 40:445-456. ["Internet"], [acceso 09 de junio de 2021]. Disponible en: https://www.minervamedica.it/en/journals/ cardiovascular-surgery/article.php?cod=R37Y1999N03A0445

2. Ballantyne JC, Carr DB, deFerranti S, y cols. The comparative effects of postoperative analgesic therapies on pulmonary out-come: cumulative meta-analyses of randomized, controlled trials. Anesth Analg. 1998; 86:598-612. https://doi. org/10.1213/00000539-199803000-00032

3. Batchelor T, Rasburn N, Abdelnour-Berchtold E, y cols. Guidelines for enhanced recovery after lung surgery: recommendations of the Enhanced Recovery After Surgery (ERAS $\otimes)$ Society and the European Society of Thoracic Surgeons (ESTS). Eur J Cardiothorac Surg. 2019; 55(1):91-115. https://doi.org/10.1093/ejcts/ezy301

4. Azad SC, Groh J, Beyer A, Schneck D, Dreher E, Peter K. Continuous peridural analgesia vs patient - controlled intravenous analgesia for pain therapy after thoracotomy. Anaesthesist. 2000; 49: 9-17. ["Internet"], [acceso 9 de junio de 2021]. https://doi. org/10.1007/s001010050003

5. Forero M, Adhikary SD, Lopez H, y cols. The erector spinae plane block: a novel analgesic technique in thoracic neuropathic pain. 
Reg Anesth Pain Med. 2016; 41:621-7. https://doi.org/10.1097/ AAP.0000000000000451

6. Forero M, Rajarathinam M, Adhikary S, y cols. Erector spinae plane (ESP) block in the management of post thoracotomy pain syndrome: A case series. Scand J Pain 2017; 17:325-9. https:// doi.org/10.1016/j.sjpain.2017.08.013

7. Schwartzmann A, Peng P, AntunezMaciel M, Alcarraz P, González $X$, Forero $M$. A magnetic resonance imaging study of local anesthetic spread in patients receiving an erector spinae plane block. Can J Anaesth. 2020; 67 (8): 942-948. https://doi. org/10.1007/s12630-020-01613-8

8. Ivanusic J, Konishi Y, Barrington MJ. A Cadaveric Study Investigating the Mechanism of Action of Erector Spinae Blockade. Regional Anesthesia and Pain Medicine. 2018; 43: 567-571. https:// doi.org/10.1097/AAP.0000000000000789

9. Kendall M, Alves L, Traill L, De Oliveira $G$. The effect of ultrasound-guided erector spinae plane block on postsurgical pain: a meta-analysis of randomized controlled trials. BMC Anesthesiology. 2020; 20:99. https://doi.org/10.1186/s12871-020-01016-8

10. Huang W, Wang W, Xie W, Chen Z, Liu Y. Erector spinae plane block for postoperative analgesia in breast and thoracic surgery: A systematic review and meta-analysis. J Clin Anesth. 2020; 66:109900. https://doi.org/10.1016/j.jclinane.2020.109900

11. Yao Y, Fu S, Dai S, y cols. Impact of ultrasound-guided erector spinae plane block on postoperative quality of recovery in videoassisted thoracic surgery: A prospective, randomized, controlled trial. J Clin Anesth. 2020; 63: 109783. https://doi.org/10.1016/j. jclinane.2020.109783

12. Wang Q, Zhang G, Wei S, He Z, Sun L, Zheng H. Comparison of the Effects of Ultrasound-guided Erector Spinae Plane Block and Wound Infiltration on Perioperative Opioid Consumption and Postoperative Pain in Thoracotomy. J Coll Physicians Surg Pak. 2019; 29(12):1138-1143. https://doi.org/10.29271/ jcpsp.2019.12.1138 